\title{
HILGARDIA
}

A Journal of Agricultural Science Published by the California Agricultural Experiment Station

CONTENTS

THE EFFECT OF CERTAIN ADDED MATERIALS ON BORDEAUX MIXTURE INTHE CONTROL OF PEACH BLIGHT AND LEAF CURL EDWARD E. WIISON

PHYTOPHTHORA CINNAMOMI AND WET SOIL IN RELATION TO THE DYING-BACK OF AVOCADO TREES

VINCENT A. WAGER

PYTHIACEOUS FUNGI ON CITRUS VINCENT A. WAGER

UNIVERSITY OF CALIFORNIA - BERKELEY, CALIFORNIA 


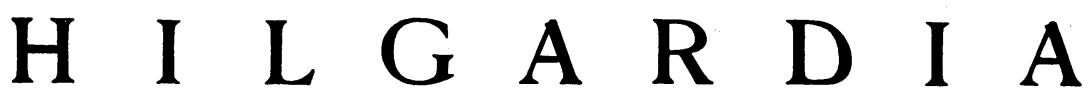

A Journal of Agricultural Science Published by

the California Agricultural Experiment Station

VoL. 14

AUGUST, 1942

No. 9

\section{THE EFFECT OF CERTAIN ADDED MATERIALS ON BORDEAUX MIXTURE IN THE CONTROL OF PEACH BLIGHT AND LEAF CURL ${ }^{1}$}

\author{
EDWARD E. WILSON ${ }^{2}$
}

\section{INTRODUCTION}

IN THE STANDARD method for controlling peach blight, caused by Coryneum Beijerinckii Oud., bordeaux mixture (10-10-100) is applied to the trees after all leaves are off in the autumn, but before the winter rains begin. Since this is the only application given to protect the twigs against blight during the following three or four months, when heavy and prolonged rains occur, and since this application is also expected to prevent leaf curl caused by Taphrina deformans, success of the control is largely dependent upon resistance of the fungicide deposit to dissipation by atmospheric agencies.

In earlier trials (12), ${ }^{8}$ bordeaux to which 4 per cent of a dormant petroleum-oil emulsion was added, proved more weather-resistant than bordeaux without oil-results that were in agreement with those of Winston, Bowman, and Yothers (14). In 1936-37 and 1937-38, therefore, further trials were undertaken to determine whether smaller amounts of petroleum oil would reduce the loss of bordeaux from peach twigs as effectively as 4 per cent does, and whether other materials such as cottonseed oil and bentonite were useful in this respect.

The effect of petroleum oil on the toxicity of bordeaux to spores of Coryneum Beijerinckii (13), and on the spray's "retention"“ and "coverage" qualities were also studied.

\footnotetext{
${ }^{1}$ Received for publication November 13, 1941.

Assistant Professor of Plant Pathology and Associate Plant Pathologist in the Experiment Station.

"Italic numbers in parentheses refer to "Literature Cited" at the end of this paper.

- The literature is not unanimous regarding designation of the qualities that determine the efficacy of insecticides and fungicides. Thus the amount of spray per unit of surface remaining after application, is called "initial spray deposit" (16), "deposition" ( 8 ), "initial adhesiveness" (9), and "retention" (2); the distribution of the material over the surface is "coverage" $(3)$; and the resistance to weathering is "adherence" (10) or "tenacity" (2). Horsfall, Heuberger, Sharvelle, and Hamilton (8) designate as "fungicidal value" that ability of the material to prevent spore germination; but the present writer prefers "toxicity" or "toxicological value."
} 


\section{EFFECT OF PETROLEUM OIL, COTTONSEED OIL, AND BENTONITE ON TENACITY OF BORDEAUX}

Each spray treatment was given in the autumn to four randomized plots, each containing four Paloro peach trees of fairly uniform size. The treatments were bordeaux mixture (10-10-100) to which had been added different amounts of petroleum-oil emulsion, tank-mix petroleum oil, cottonseed oil, or bentonite. Emulsion A was a flowable-type emulsion containing 80 per cent, by weight, of a petroleum oil of 102 seconds Saybolt viscosity and 70 per cent unsulfonated residue. Tank-mix oil was a petroleum oil similar to that in emulsion A emulsified with blood albumin just before it was added to the spray tank. Emulsion B was a paste-type emulsion containing 82 per cent, by weight, of a petroleum oil of 96 seconds viscosity and 94 per cent unsulfonated residue. The cottonseed oil was a commercial grade. The bentonite was a natural product containing some magnesium oxide.

As soon as the spray dried, 200- to 250-gram samples of twigs produced during the past summer were collected and weighed. The twigs were then cut into convenient lengths, placed in glass jars, and shaken for 10 minutes with $500 \mathrm{ce}$ of nitric acid water (20 ec nitric acid, of $1.42 \mathrm{sp}$. gr., per liter). The wash water was filtered and tested for copper by the sodium diethyl dithiocarbamate method ${ }^{5}$ that Callan and Henderson (1) described.

Other collections were made after several inches of rain had fallen (December or January) and again just before the buds swelled in the spring (February). The amounts of copper on these samples were the basis for determining the weather resistance or tenacity of the bordeaux.

Experiments of 1936-37.-Sprays were applied November 20, 1936, well before the first major wave of twig infection by Coryneum which was initiated during rains falling between December 20 and 28. The season was marked by recurrent attacks of the disease during January, February, and March-particularly severe being a wave initiated in early March.

According to the November 20 analyses (table 1), considerable variability existed in the initial amount of copper deposited by the various treatments. These data, together with those secured in other years, will be discussed later.

\footnotetext{
${ }^{5}$ In the present work the results obtained by the Callan and Henderson method were consistently higher, by about 3 per cent, than results obtained by the electrometric method, but compared more favorably with those secured by the iodometric method. Though the last-named method cannot be used when very small amounts of copper are to be determined, it is a valuable referee for standards used in colorimetric tests.
} 


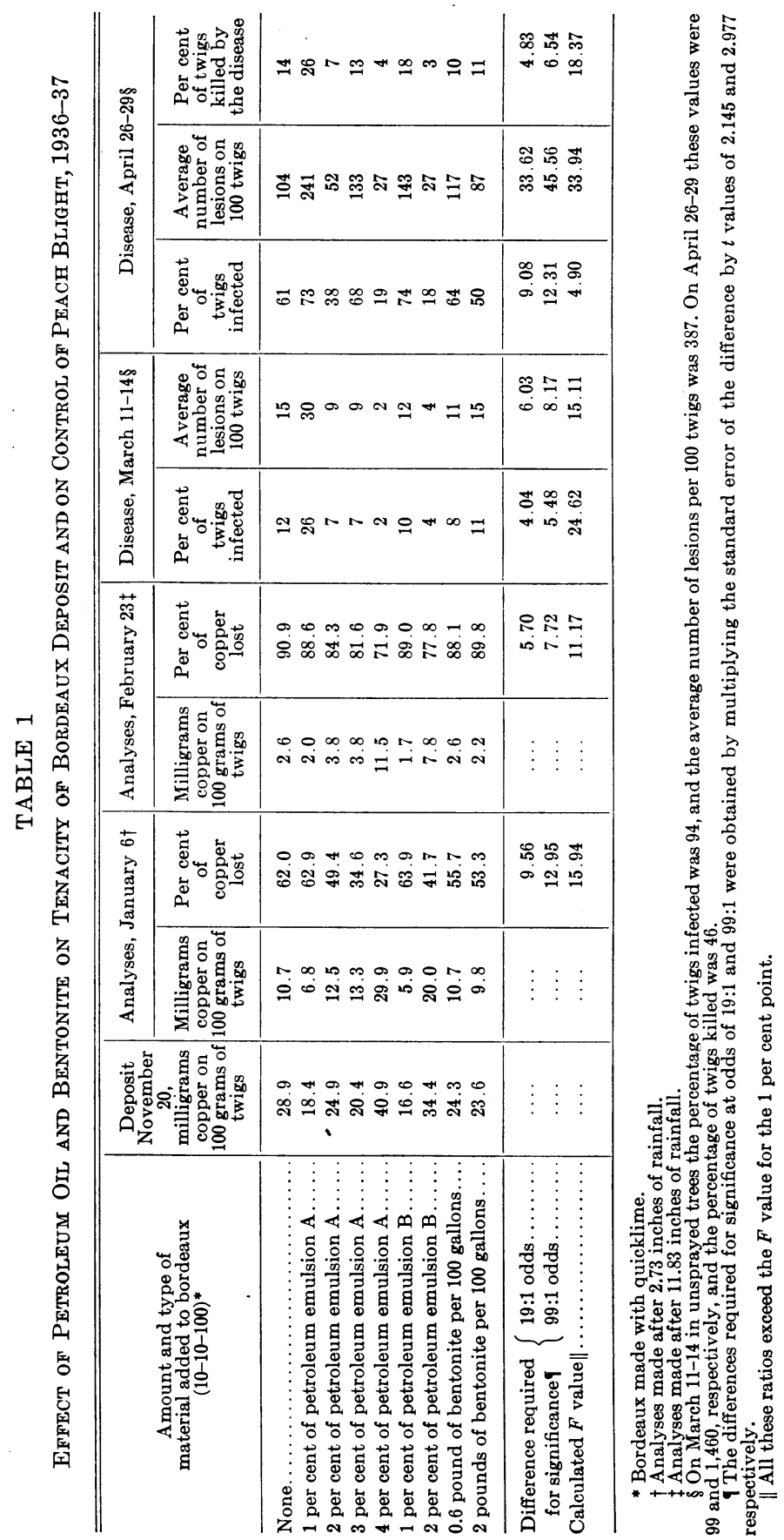


After 2.73 inches of rain had fallen, analyses (January 6) revealed marked differences in rate of copper loss from trees receiving the various treatments. Apparently neither of the two types of oil (emulsion A and emulsion B) when used at the rate of 1 per cent influenced the tenacity of bordeaux. The February 23 analyses further emphasize this point, the percentage of copper lost being practically the same for bordeaux alone, bordeaux with 1 per cent of emulsion A, and bordeaux with 1 per cent of emulsion B. Neither in the January 6 nor in the February 23 analyses was bentonite found significantly to reduce the loss of copper.

Increasing the amounts of oil emulsion above the 1 per cent resulted in successively improved tenacity. In this respect, 4 per cent of emulsion A was significantly more efficient than 2 or even 3 per cent, and 2 per cent of emulsion B produced greater tenacity than 1 per cent.

January 6 analyses showed that most treatments still retained considerable amounts of copper. Exceptions were bordeaux plus 1 per cent of emulsion A and bordeaux plus 1 per cent of emulsion B, where the low amount of residue was influenced by low initial deposits. The depletion of the spray coating on trees receiving these two treatments is reflected in the control data obtained March 11-14. Bordeaux plus 1 per cent of emulsion A, in particular, was showing signs of losing its effectiveness at this time. The possibility that the addition of oil might have affected the initial deposit will be discussed later.

According to data on disease conditions in unsprayed trees, as given in the footnote of table 1, most other treatments gave good control up to March 11-14. During early March, however, rains initiated abundant infection; the resulting lesions appeared after March 14, and between this date and April 26-29 the number of lesions on unsprayed trees more than tripled. Bordeaux and bordeaux plus 1 per cent of emulsion A or of $B$ gave poor control during this period, whereas bordeaux plus 2 or 4 per cent of emulsion A and bordeaux plus 2 per cent of emulsion B proved much more efficient. The poor control obtained with bordeaux plus 3 per cent of emulsion A cannot be explained fully by the data at hand. The low initial deposit may have been responsible to some extent, though the amount of copper remaining on February 23 was the same as that for 2 per cent of emulsion A. The 3 per cent of emulsion treatment controlled well up to the early March infection period, but failed thereafter.

Bentonite did not significantly influence the control one way or the other.

On February 1, 1937, bordeaux with and without oil was applied to almond trees. As no blight of consequence developed in this orchard, 
TABLE 2

Relation of Petroleum Oil Emulsion to the Retention and Tenacity of Bordeaux Deposit on Almond Twigs, 1937

\begin{tabular}{|c|c|c|c|}
\hline Amount of oil added to bordeaux $(10-10-100)$ & $\begin{array}{l}\text { Analyses, } \\
\text { February 1; } \\
\text { milligrams } \\
\text { of copper } \\
\text { per } 100 \text { grams } \\
\text { of twigs* }\end{array}$ & $\begin{array}{l}\text { Analyses, } \\
\text { February 14; } \\
\text { milligrams } \\
\text { of copper } \\
\text { per } 100 \text { grams } \\
\text { of twigs } \dagger\end{array}$ & $\begin{array}{l}\text { Per cent of } \\
\text { copper lost }\end{array}$ \\
\hline 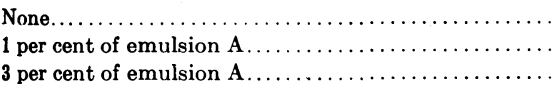 & $\begin{array}{l}22.1 \\
24.1 \\
30.0\end{array}$ & $\begin{array}{r}6.4 \\
4.7 \\
16.9\end{array}$ & $\begin{array}{l}71.1 \\
81.5 \\
44.7\end{array}$ \\
\hline $\begin{array}{l}\text { Difference required for significance: } \neq\left\{\begin{array}{c}19: 1 \text { odds.... } \\
99: 1 \text { odds..... }\end{array}\right. \\
\text { Calculated } F \text { value } \$ \ldots \ldots \ldots \ldots \ldots \ldots \ldots \ldots \ldots \ldots \ldots\end{array}$ & $\begin{array}{r}3.65 \\
5.24 \\
12.85\end{array}$ & $\begin{array}{r}3.90 \\
5.60 \\
29.43\end{array}$ & $\begin{array}{l}12.7 \\
18.3 \\
23.58\end{array}$ \\
\hline
\end{tabular}

- Spray was applied February 1 and samples for these analyses were made immediately after it dried. + Between February 1 and February 14, 5.19 inches of rain fell.

$\$$ The differences required for significance at odds of $19: 1$ and $99: 1$ were obtained by multiplying the standard error of the difference by $t$ values of 2.262 and 3.250 respectively.

$\$$ All these ratios exceed the $F$ value for the 1 per cent point.

\section{TABLE 3}

EFfect of Different Supplements on the Control of Peach Leaf CURL by BORDEAUX MIXTURE

\begin{tabular}{|c|c|c|c|}
\hline $\begin{array}{l}\text { Amount and type of material } \\
\text { added to bordeaux } \\
(10-10-100), 1936-37^{*}\end{array}$ & $\begin{array}{l}\text { Per cent } \\
\text { of leaves } \\
\text { diseased } \dagger\end{array}$ & $\begin{array}{l}\text { Amount and type of material } \\
\text { added to bordeaux } \\
(10-10-100), 1937-38^{*}\end{array}$ & $\begin{array}{l}\text { Per cent } \\
\text { of leaves } \\
\text { diseased } \dagger\end{array}$ \\
\hline Unsprayed. & 56 & Unsprayed. & 49 \\
\hline None........ & 2 & None................ & 0.4 \\
\hline 1 per cent of petroleum emulsion $A$.. & 8 & 1 per cent of petroleum emulsion $A$. . & 2 \\
\hline 2 per cent of petroleum emulsion A.. & 0.4 & 3 per cent of petroleum emulsion $A$. & 0.2 \\
\hline 3 per cent of petroleum emulsion A.. & 2 & 1 per cent of petroleum tank-mix oil... & 1 \\
\hline 4 per cent of petroleum emulsion $A$.. & 0.4 & 3 per cent of petroleum tank-mix oil... & 0.1 \\
\hline 1 per cent of petroleum emulsion $B .$. . & 2 & 1 per cent of cottonseed oil ........... & 1 \\
\hline 2 per cent of petroleum emulsion B... & 0.5 & & \\
\hline 0.6 pound of bentonite per 100 gallons. & 0.7 & & \\
\hline 2 pounds of bentonite per 100 gallons. . & 0.5 & & \\
\hline
\end{tabular}

- Sprays were applied in 1936-37 on November 20; in 1937-38 on November 23.

† Observations on leaf infection made in late April. The percentage of leaves infected was determined by counting the leaves on 20 randomly selected twigs in each tree.

evidence on control was not obtained. The data on copper analyses (table 2), however, further confirmed the results secured on peaches, in that 1 per cent of oil emulsion had no effect on bordeaux tenacity, whereas 3 per cent materially increased it.

As peach-leaf curl developed abundantly on unsprayed trees, counts were made on the percentage of leaves diseased in trees receiving the treatments listed in table 1 . The results (table 3 ) showed no marked failure of any treatment to control this disease, though in one of the 


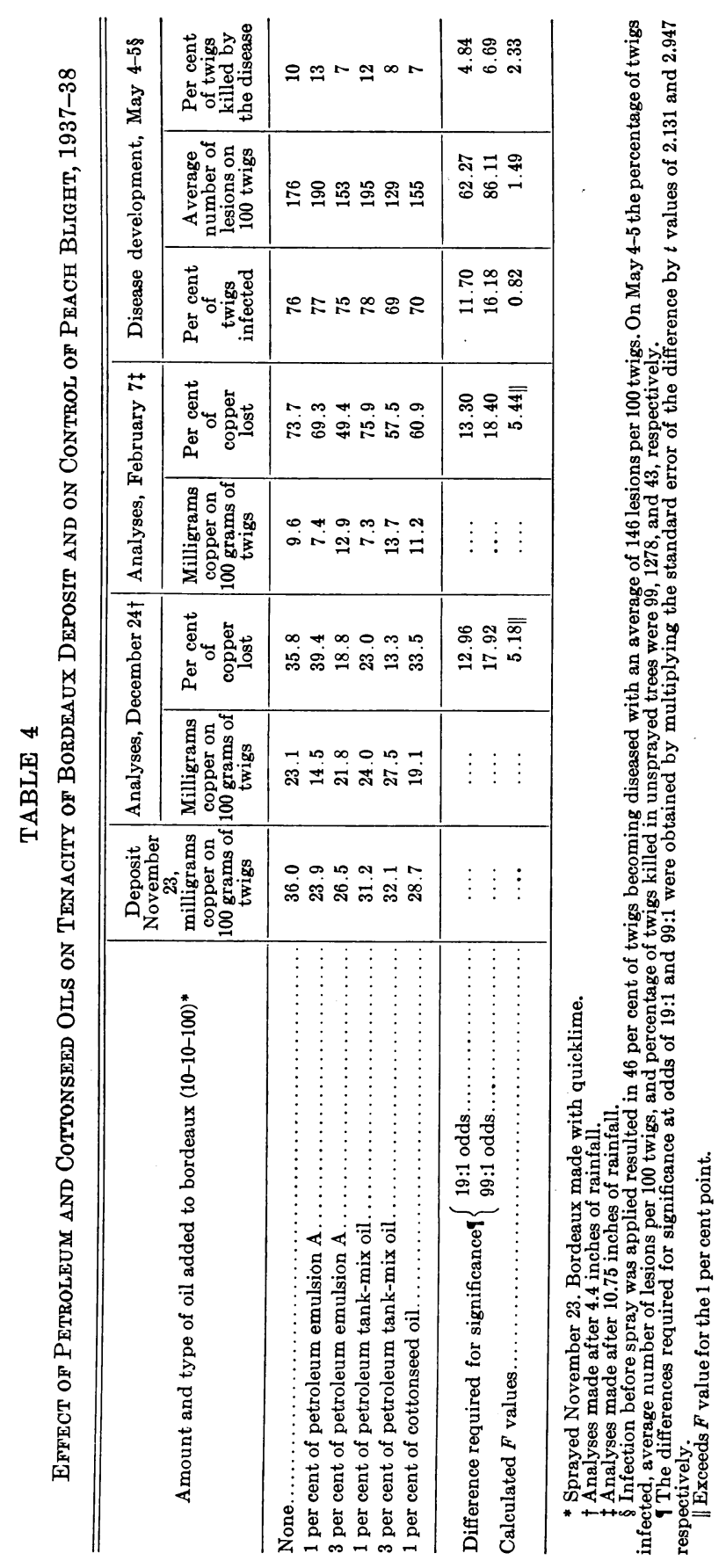


four replications of bordeaux plus 1 per cent of emulsion A the incidence of the disease was rather high.

Experiments of 1937-38.-Twig infection was initiated in mid-November, 1937, and in consequence a considerable part of the disease recorded in the final counts on May 4-5, 1938, was present in the trees when the sprays were applied on November 23. The major part of the twig lesions appeared during November and December; none appeared during January, and only a few in February. Therefore, disease development in this season differed markedly from that in 1936-37, when lesions were appearing throughout the winter and a particularly large number appeared in early March. It is apparent that the problem of control in 1937-38 also differed from that in 1936-37, although the incidence of the disease was the same in both years (footnotes to tables 1 and 4).

Sprays were applied on November 23, and samples collected on the same day were analyzed for initial deposits (table 4). Collection of samples on December 24 (after 4.4 inches of rainfall) indicated, as in 1936-37, that 1 per cent of oil emulsion A did not affect bordeaux tenacity ; but 3 per cent increased the tenacity considerably. The same general relation between these treatments and bordeaux without oil was maintained up to February 7, during which time an additional 6.35 inches of rain fell. According to the December 24 analyses 1 per cent of tankmix oil appeared to favor bordeaux tenacity, but the February 7 analyses revealed no advantage over bordeaux without oil. Three per cent of tank-mix oil was about as efficient as 3 per cent of emulsion $A$ in preventing copper loss. One per cent of cottonseed oil appeared, in the February 7 analyses, to have decreased the loss only slightly if any.

As was mentioned at the beginning of this section, twig lesions were developing in the trees at the time the sprays were applied. Two days after application an average of 146 lesions per 100 twigs were present on 46 per cent of the twigs. To show more clearly the control situation, the increment of 146 lesions per 100 twigs is deducted from the results in table 4 and is arranged with the data on copper residues as of February 7 (table 5 ). Some correlation between the amount of copper remaining on the twig and the number of new lesions is thus shown. The copper residues on this date have the same general relation to the percentage of twigs killed, although such differences cannot be considered statistically significant (table 4).

When, therefore, deductions are made for the disease developing before sprays were applied, all treatments are seen to give excellent control. The reason for this high efficiency is the manner in which disease 
developed : the major attacks came early in the winter at a time when the spray film on the trees was new, instead of in late winter after weathering had reduced it, as in 1936-37.

TABLE 5

Relation of amount of Copper Remaining on Twigs Near the End of the Season to the Control of Peach Blight Obtained WITH BoRdeauX AND BoRdeaUX PLUS OIL, 1937-38

\begin{tabular}{|c|c|c|}
\hline $\begin{array}{l}\text { Amount and type of material added } \\
\text { to bordeaux }(10-10-100)^{*}\end{array}$ & $\begin{array}{c}\text { Milligrams } \\
\text { of copper } \\
\text { per } 100 \text { grams } \\
\text { of twigs, } \\
\text { February } 7\end{array}$ & $\begin{array}{c}\text { Average } \\
\text { number } \\
\text { of lesions } \\
\text { (per } 100 \text { twigs) } \\
\text { developing } \\
\text { after spraying } \dagger\end{array}$ \\
\hline Unsprayed $\ldots \ldots \ldots \ldots \ldots \ldots \ldots$ & $\ldots$ & 1,132 \\
\hline None $\ldots \ldots \ldots \ldots \ldots \ldots \ldots$ & 9.6 & 30 \\
\hline 1 per cent of oil emulsion $A \ldots \ldots$. & 7.4 & 44 \\
\hline 3 per cent of oil emulsion $A \ldots$. & 12.9 & 7 \\
\hline 1 per cent of tank-mix oil.. & 7.3 & 49 \\
\hline 3 per cent of tank-mix oil.. & 13.7 & 0 \\
\hline
\end{tabular}

- Sprayed November 23. Bordeaux made with quicklime.

t Infection before the spray was applied on November 23 resulted in an average of 146 lesions per 100 twigs. The values in this column were obtained by deducting this number from the average number of lesions per 100 twigs reported in table 4.

\section{TABLE 6}

Improvement in the Tenacity of Bordeaux Deposit and the Control of Peach Blight When Petroleum-Oir Emulsion Was Added, 1937-38*

\begin{tabular}{c|c|c|c|c}
\hline \hline Treatmentt & $\begin{array}{c}\text { Deposit } \\
\text { November 25, } \\
\text { milligrams } \\
\text { copper on } \\
\text { 100 grams } \\
\text { of twigs }\end{array}$ & $\begin{array}{c}\text { February 27, } \\
\text { milligrams } \\
\text { copper on 100 } \\
\text { grams of twigs }\end{array}$ & $\begin{array}{c}\text { Per cent of } \\
\text { copper lost }\end{array}$ & $\begin{array}{c}\text { Per cent } \\
\text { of twigs killed } \\
\text { by the disease } \\
\text { (April 15) }\end{array}$ \\
\hline $\begin{array}{c}\text { Bordeaux }(10-10-100) \ldots \ldots \ldots \ldots \ldots \ldots \\
\text { Bordeaux }(10-10-100) \text { plus 4 per cent } \\
\text { of emulsion A....................... }\end{array}$ & 29.3 & 6.4 & 14 \\
\hline
\end{tabular}

* From data secured in a commercial orchard.

† Spray applied November 25 . Bordeaux was prepared with hydrated lime, which was soaked 1 to 2 hours before using.

Some additional studies were made in a commercial orchard where bordeaux (10-10-100) alone and bordeaux (10-10-100) plus 4 per cent of oil emulsion A were applied. These studies (table 6) further demonstrated the increased tenacity attending the addition of a large amount of oil to bordeaux. Some improvement in control was also apparent.

As in 1936-37, leaf curl developed abundantly in unsprayed plots in the experimental orchard. No significant difference in control attended the various treatments listed in table 4 ; all gave excellent protection (table 3 ). 


\section{EFFECT OF PETROLEUM-OIL EMULSION ON THE AMOUNT OF BORDEAUX DEPOSITED ON A SURFACE}

The unsatisfactory control of twig infection in 1936 (table 1) which attended the applications of bordeaux containing 1 per cent of oilemulsion $\mathrm{A}$ and of $\mathrm{B}$ was attributed, in part at least, to the low initial

TABLE 7

EFfect of Petroleum and Cottonseed OILs on the Retention of Bordeatux Mixture by Peach Twigs

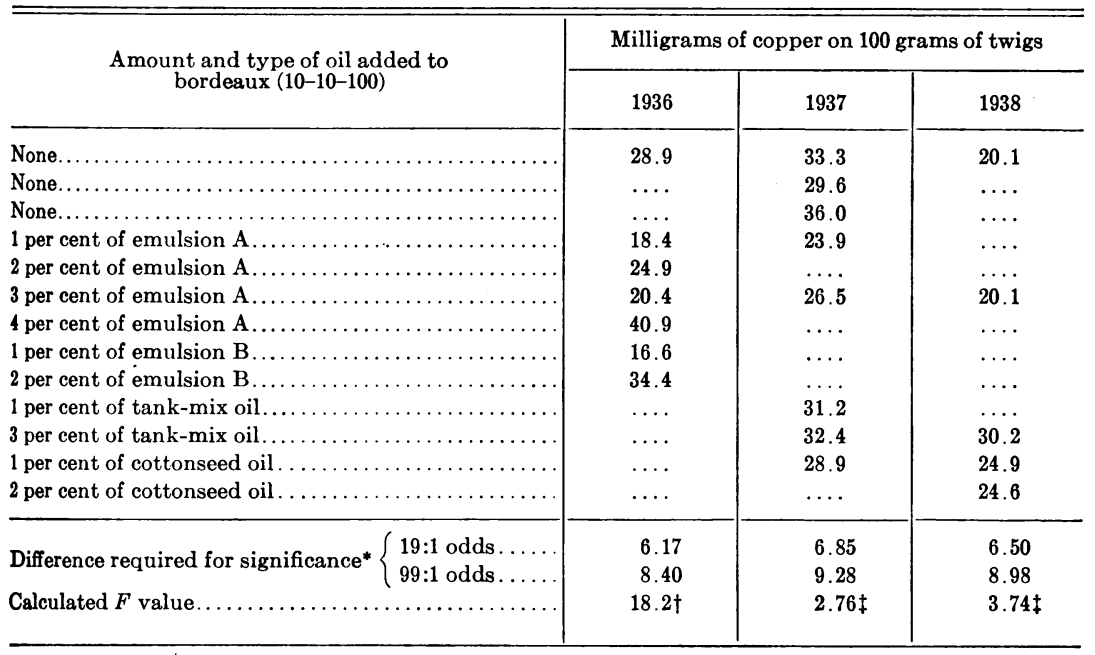

* Differences required for significance at odds of 19:1 and 99:1 were obtained by multiplying the standard error of the difference by $t$ values respectively as follows: 1936, 2.08 and $2.831 ; 1937,2.064$ and $2.797 ; 1938,2.131$ and 2.947 .

Exceeds $F$ value for the 1 per cent point.

$\$$ Exceeds $F$ value for the 5 per cent point.

deposit of the fungicide. A low deposit and unsatisfactory control were also recorded for the treatment containing 3 per cent of emulsion A: the amount of copper residue remaining on the trees in late February, shortly before the infection period that proved critical, was not materially lower than in treatments that remained effective during the critical period. On the other hand, treatments that controlled twig infection most satisfactorily-bordeaux containing 4 per cent of emulsion A and 2 per cent of emulsion B-gave initial deposits considerably higher than other treatments.

Whereas analysis of variance (table 7) shows that in 1936 the initial deposit of the bordeaux with 1 per cent of oil was significantly lower than that of bordeaux without oil, neither 1 per cent nor 3 per cent of 
emulsion A significantly reduced deposits in 1937. In 1938, moreover, 3 per cent of emulsion A did not affect copper deposits. The treatment containing 3 per cent of tank-mix oil, on the contrary, deposited more copper than bordeaux without oil in 1938, but not in 1937. Considering these variations and the character of the results secured on almond in 1936 (table 2), at which time bordeaux plus 3 per cent of emulsion A deposited higher amounts of copper than bordeaux without oil, one can draw no definite conclusions regarding the effect of oil on initial deposits.

To obtain further information under laboratory conditions, a small spray applicator was constructed. This consisted of a framework at one end of which was attached a no. 16 De Vilbiss atomizer in a horizontal position; at the other end, 16 inches from the nozzle of the atomizer, a clamp held the object to be sprayed. A rubber tube led from the intake of the atomizer to a wide-mouthed glass jar holding the spray material. A small electric stirrer kept the spray material constantly agitated. The air blast to operate the atomizer was furnished by a laboratory pump equipped with an adjustable pressure-release valve. In order quickly to begin and end application without disturbing the delivery of spray from the atomizer, the nozzle was enclosed in a metal cup with an aperture in line with the stream of spray. This aperture was opened and closed by a shutter.

Though a number of improvements, such as that suggested by Horsfall, Heuberger, Sharvelle, and Hamilton (8) to control humidity, could be made in this apparatus, it was proved well suited to the purpose, which was to apply two or three different materials within a few minutes of each other.

Though Horsfall and his associates suggested as a standard surface that pyroxylin (cellulose nitrate) be dissolved in butyl acetate and deposited on glass, the present writer prepared the cellulose nitrate in the laboratory, and dissolved it in three parts of ether to one of alcohol. The surfaces were prepared by dipping microscope slides into this solution and standing the slides vertically in a dust-free atmosphere to dry for 24 hours.

Since the applicator was found to deliver bordeaux and bordeaux plus oil (hereafter called "oil-bordeaux" except where the type of oil is specified) at the same rate, some definite time or stage had to be established as an end-point in application. Evans and Martin (2) had applied the spray until the liquid began to run down the surface. Hoskins and Ben-Amotz (9), on the other hand, ended application after a measured amount of liquid had drained from the surface. In the present work certain considerations guided the decision to end application at the two 
following stages: (1) at the point just before the liquid began to run down the surface (runoff stage), and (2) when about 1 cc of liquid had drained from the surface (drip stage). The considerations were as follows : assuming equal delivery of two materials from the atomizer, it is evident that at least during the early stages of application the liquid will be deposited at equal rates. If, therefore, two materials were applied for the same length of time, they should deposit the same amount, pro-

TABLE 8

Effect of Petroleum-Oil Emulsion on Retention of Bordeaux Deposit by a Cellulose Nitrate Surface

\begin{tabular}{|c|c|c|}
\hline Material & $\begin{array}{c}\text { Stage } \\
\text { at which } \\
\text { application } \\
\text { was stopped }\end{array}$ & $\begin{array}{c}\text { Milligrams } \\
\text { of copper on } \\
1 \text { square centi- } \\
\text { meter of surface }\end{array}$ \\
\hline 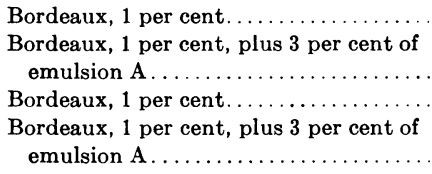 & $\begin{array}{l}\text { Runoff* } \\
\text { Runoff* } \\
\text { Drip }{ }^{*} \\
\text { Dript }\end{array}$ & $\begin{array}{l}0.0318 \\
0.0343 \\
0.0211 \\
0.0252\end{array}$ \\
\hline $\begin{array}{l}\text { Difference required for } \\
\text { significance: }\end{array}\left\{\begin{array}{l}19: 1 \text { odds...... } \\
99: 1 \text { odds...... }\end{array}\right.$ & & $\begin{array}{l}0.0019 \\
0.0026\end{array}$ \\
\hline
\end{tabular}

* Spraying was stopped when liquid showed signs of beginning to run down the surface.

† Spraying was stopped when $1 \mathrm{cc}$ (approximately) of liquid had accumulated at the lower end of the slide.

vided one material did not begin to run off the surface before the other. The runoff stage provides, therefore, a criterion to judge retention of the liquid by the surface, for if one liquid is retained less than another this liquid will require the shorter period of application to reach the runoff stage. Since in practice one cannot spray all parts of the tree to exactly the same stage as regards runoff, one should determine whether overspraying causes differences in deposits ; slides were, therefore, sprayed until about 1 cc of liquid had drained from the surface (drip stage).

Table 8 gives a typical example of spraying 10 slides each with bordeaux 1 per cent (8-8-100, approximately) and bordeaux 1 per cent plus 3 per cent of petroleum-oil emulsion $A$ to the runoff stage and to the drip stage. The deposit of oil-bordeaux is seen to be significantly higher than that of bordeaux without oil when spraying was carried to either stage. According to these data, furthermore, significantly higher amounts of copper were present on surfaces sprayed only to the runoff stage than on surfaces sprayed until drip occurred.

In five such tests, when application ended at the runoff stage, bordeaux 
with 3 per cent of oil emulsion increased deposits by 37 per cent, whereas the increase required for statistical significance at $99: 1$ odds was 21 per cent. In similar tests, when application was continued to the drip stage, oil-bordeaux increased deposits 26 per cent, whereas the increase required for significance at $99: 1$ odds was 20 per cent.

As was said earlier, the sprayer delivered oil-bordeaux and bordeaux at the same rate. If we assume, therefore, that the two materials were deposited on the surfaces at the same rate, the amount of each present at the runoff stage should differ only if the period of application necessary for producing runoff was longer with one than with the other. To test this point, one lot of bordeaux was divided into three portions. To the first was added 2 per cent of oil emulsion A; to the second, $1 / 4$ per cent of an organic spreading agent; to the third, nothing. The amount of bordeaux in all lots was adjusted to the same value by adding the requisite amount of water. In applying these materials to cellulose nitrate-covered slides, the time necessary to reach the runoff stage and the drip stage, respectively, was determined with a stop watch. On ten slides the average time necessary to produce runoff with bordeaux was 5.6 seconds ; with bordeaux plus oil emulsion A, 7.2 seconds; with bordeaux plus the organic spreading agent, 4.4 seconds. The time necessary to reach the drip stage was 13.0,16.1, and 9.7 seconds, respectively. Apparently, therefore, the time factor is one cause of the greater deposit of oil-bordeaux.

It is noteworthy that bordeaux with the spreading agent required the shortest application periods. Hockenyos and Irwin (6) found a similar situation when applying bordeaux with certain supplements to peach leaves. The present results with the spreader seem significant, furthermore, in view of the evidence by Evans and Martin (2) and Hoskins and Ben-Amotz (9) that an increase in the wetting and spreading quality of a spray material was frequently accompanied by a lower deposit. The relation of wetting and spreading to distribution of the deposit was next studied.

\section{EFFECT OF OIL ON THE DISTRIBUTION OF BORDEAUX OVER THE SURFACE}

According to studies by Evans and Martin (2), an accessory material that promotes wetting and spreading of the aqueous phase increases the uniformity with which the fungicide suspended therein is distributed over the sprayed surface. On surfaces particularly difficult for water to wet, the fungicide is deposited in unevenly distributed patches. Thus Yarwood (16) found bordeaux to be deposited on onion so unevenly as to be ineffective against the downy mildew fungus. When, however, a 
spreading agent (Penetrol) was added, the bordeaux was distributed more evenly over the leaves and, in consequence, controlled the mildew more effectively.

Since peach twigs did not furnish a satisfactory surface for studying the effects of oil on distribution of bordeaux, the following tests were conducted with cellulose nitrate on glass slides. Bordeaux and oil-bordeaux exhibited little difference in the distribution of deposit on this surface when application was stopped before the runoff started. If, however, application was continued until 1 or 2 cubic centimeters of liquid had drained from the surface, the resulting deposit of oil-bordeaux was more even and finer in grain. The reason for this difference was clearly seen in observing the manner in which the bordeaux and oil-bordeaux drained from the surface. Whereas the former flowed down the slides in a series of drops which followed irregular courses and, in consequence, left uneven deposits of the solid, the oil-bordeaux flowed down the surface as a sheet, leaving the solid more evenly distributed.

When, immediately after deposition by spraying, the individual droplets of bordeaux and oil-bordeaux were more closely studied, those of the latter were found to cover somewhat smaller areas. To examine this point further, bordeaux, with and without different combinations of oil and a wetting agent were dropped from a 1-ce pipette from a height of 1 inch upon horizontal surfaces of cellulose nitrate. After 15 minutes the diameters of the drops were measured. The volume of the drops delivered by the pipette was established for each material. Table 9 gives data on the spread of drops, the volume of drop, and a percentage relation between these two data which was obtained by dividing the diameter of the drop (resting on the surface) by its volume. The ratios thus obtained were then expressed as a percentage of the ratio for water; that is, the value for water was considered as 100.

According to table 9 the diameter of spread of 1 per cent bordeaux was 90 per cent of that of water, whereas the spread of bordeaux plus 2 per cent of oil emulsion A was 63 per cent, and bordeaux plus oil without the emulsifier was even less. The spread of oil emulsion A (used alone) was practically the same as that of bordeaux plus oil emulsion A.

The organic spreading agent more than doubled the spread of water, noticeably increased the spread of bordeaux, and overcame the tendency for oil to restrict the spread of bordeaux. Since the amount of organic spreading agent was much more than the amount of emulsifier introduced into the spray with oil emulsion $A$, this is not a fair comparison of the spreading efficiency of the two. The differences in the results secured serve, however, to emphasize the relatively small influence the 
emulsifier in emulsion A had on the spreading of oil-bordeaux under conditions favorable to spreading.

The conditions of these tests favored spread ${ }^{6}$ because the time interval elapsing between deposition and measurement of the drops allowed for possible extension of the liquid over the surface by capillarity. In this respect, among others, the conditions of the tests differ from those exist-

\section{TABLE 9}

EFFect of Different Materials on the Area Covered by a Drop of Bordeaux Mixture on a Cellulose Nitrate SURface

\begin{tabular}{|c|c|c|c|}
\hline Material* & $\begin{array}{c}\text { Mean diameter } \\
\text { of drops } \\
\text { on surface, } \\
\text { millimeters } \dagger\end{array}$ & $\begin{array}{l}\text { Volume } \\
\text { of drops, } \\
\text { cubic } \\
\text { centimeters }\end{array}$ & $\begin{array}{l}\text { Percentage } \\
\text { relations } \\
\text { between } \\
\text { data }\end{array}$ \\
\hline 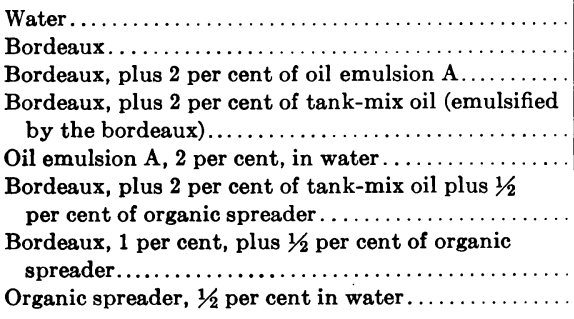 & $\begin{array}{r}10.1 \\
8.9 \\
6.1 \\
5.8 \\
6.4 \\
6.5 \\
7.8 \\
12.6\end{array}$ & $\begin{array}{l}0.051 \\
0.050 \\
0.049 \\
0.050 \\
0.050 \\
0.033 \\
0.038 \\
0.027\end{array}$ & $\begin{array}{r}100 \\
90 \\
63 \\
59 \\
65 \\
99 \\
104 \\
236\end{array}$ \\
\hline $\begin{array}{l}\text { Difference required for significance: }\left\{\begin{array}{l}19: 1 \text { odds } \ldots \ldots \\
99: 1 \text { odds } \ldots \ldots\end{array}\right. \\
\text { Calculated } F \text { value } \ldots \ldots \ldots \ldots \ldots \ldots \ldots \ldots \ldots \ldots \ldots \ldots\end{array}$ & $\begin{array}{r}0.7 \\
0.9 \\
99.6\end{array}$ & $\begin{array}{l}\cdots \cdots \\
\cdots \cdots \\
\cdots \cdots\end{array}$ & $\begin{array}{l}\cdots \\
\cdots \\
\cdots\end{array}$ \\
\hline
\end{tabular}

* Bordeaux was prepared from 1 part of copper sulfate, 1 part of lime, and 100 parts of water, approximately 8-8-100.

$\dagger$ Measurements made 15 minutes after the material was deposited on the slide.

The percentages were calculated as follows: By dividing the diameter of the drop on the surface by its volume, a ratio was obtained for each set of values. For water the ratio was considered 100 per cent; the others were referred to this basis.

ing under actual spray application. Where spray is applied, the liquid on the surface is constantly being disturbed by oncoming spray and, in consequence, the surface forces that determine its wetting and spreading properties do not attain equilibrium. The force of impact of spray with the surface is a second factor not of particular influence in these tests. Gravity in its tendency to pull the spray droplets down the vertical or inclined surface is a third factor that was not operative. Therefore, one cannot conclude that the same differences in the area covered by the individual droplets of bordeaux and oil-bordeaux will necessarily occur under actual spraying conditions. Some difference does exist, as was indicated by the observations, noted earlier, that the areas covered by

\footnotetext{
${ }^{6}$ For a discussion of the wetting and spreading properties of liquids, and the influence of time on the expression of these properties, see Evans and Martin (2), Hensill and Hoskins (5), and Hoskins and Ben-Amotz (9).
} 
individual droplets of the two types of bordeaux deposited by the applicator were not the same, those of oil-bordeaux being smaller than those of bordeaux. Whether an increase in the pressure of application would modify these differences is not known. A greater force of impact of spray with surface might force the droplets of oil-bordeaux to flatten out and cover a greater area even though the liquid did not wet this area. Droplets of bordeaux would probably be affected likewise.

Although the effect of impact pressure on spreading of the spray droplets was not studied, the following tests indicate that within the interval between deposition and drying, neither bordeaux nor oil-bordeaux spreads beyond the area they occupied when they were deposited. Drops were placed on horizontal cellulose nitrate surfaces in the manner described earlier. Their diameters were measured upon deposition and again after most of the water had disappeared. Whereas neither bordeaux nor bordeaux containing 3 per cent of oil emulsion A spread during this interval, bordeaux containing $1 / 4$ per cent of the organic wetting agent spread appreciably. Thus it would seem that the difference between the area covered by a given volume of bordeaux and oil-bordeaux was not due to difference in their spreading properties, but was probably (in part at least) the result of differences in wetting properties. A second factor, viscosity, also might have been partially responsible, but this phase was not studied.

The behavior of the solid (bordeaux precipitate) and oil phases was also observed during the drying of films deposited by the applicator. Whereas the solid phase showed no tendency to rearrange itself during this process, the oil behaved as follows: In freshly deposited drops the oil was visible as small globules enmeshed in the bordeaux precipitate. They retained their shape until most of the water had evaporated, but then broke from the emulsified state and spread over and among the solid particles. That the oil also deposited as a film on the surface was shown by the fact that an oil coating was left on the slides after the bordeaux had been removed with a weak acid.

\section{EFFECT OF OIL ON TOXICITY OF BORDEAUX TO FUNGUS SPORES}

If the release of soluble copper from the bordeaux film is requisite to toxic action, anything altering this release will affect toxicity. Holland, Dunbar, and Gilligan (7) believed that attempts to increase bordeaux tenacity might impair its toxicity. Goldsworthy and Green (4) claimed that some accessory materials rendered the particles of certain copper fungicides impervious to external solubilizing agencies. 
Observations presented in the previous section suggest that when an oil-bordeaux film dries, the oil coats the bordeaux precipitate. At the time spray is applied the oil is dispersed as droplets among the bordeaux particles, but upon disappearance of water from the film the oil droplets were seen to break and spread over and among the bordeaux particles. The question of whether this coating of oil affected toxicity of bordeaux to fungus spores was studied.

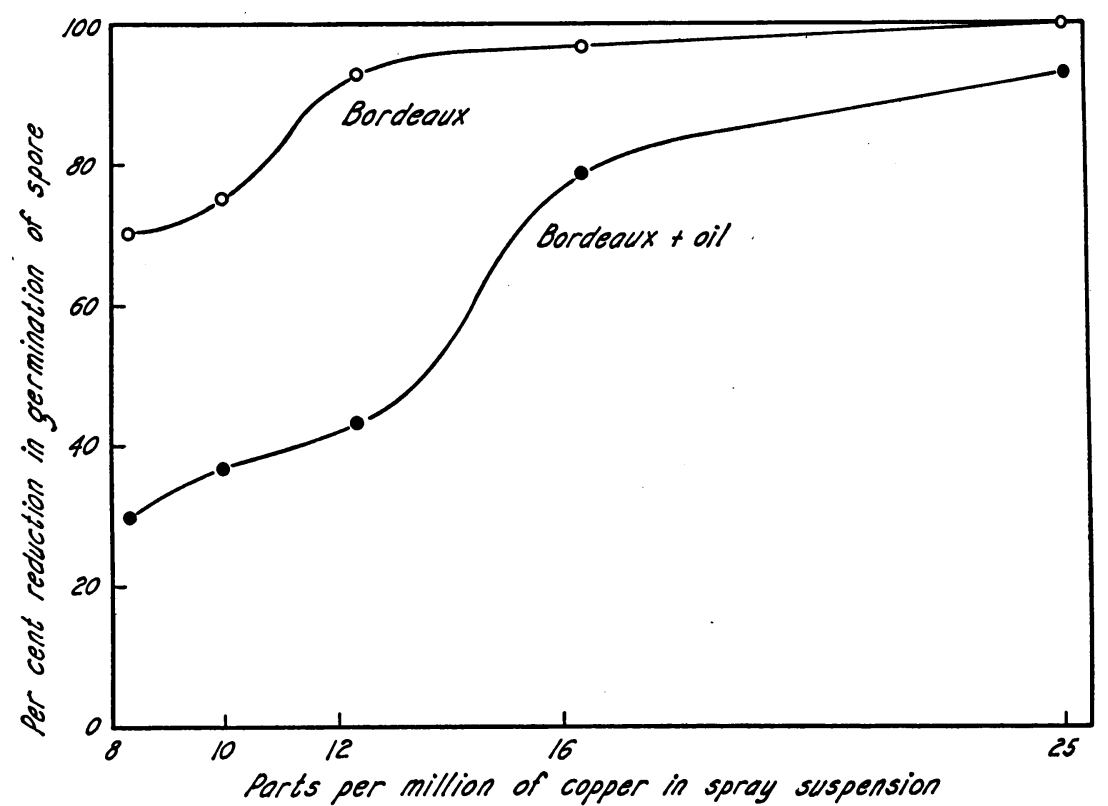

Fig. 1.-Effects of diluting bordeaux and bordeaux plus 3 per cent of oil on the toxicity of their dried films to germination of spores of Coryneum Beijerinckii. Undiluted (8-8-100) bordeaux of both types proved highly toxic, but with dilution oilbordeaux was reduced in toxicity more rapidly than bordeaux.

As the tests showed, freshly prepared films of bordeaux (8-8-100) and of bordeaux (8-8-100) plus 3 per cent of oil have such uniformly high toxicity to spores as to make comparisons at this concentration impossible. Spores of neither Coryneum Beijerinckii nor Sclerotinia fructicola germinated when placed over the two types of dried films. The two types of bordeaux, therefore, were diluted successively; samples of each dilution were dried on glass slides; and spores of the two fungi suspended in sterile, distilled water were placed over the dried films. Figure 1 represents the percentages of reduction in germination of the spores of Coryneum Beijerinckii, plotted against parts per million of copper in the bordeaux suspension. Bordeaux was consistently more 
effective in reducing germination than oil-bordeaux, both with Coryneum Beijerinckii and with Sclerotinia fructicola.

A second type of experiment was performed as follows : Bordeaux and bordeaux plus 2 and 4 per cent of oil emulsion A were diluted to contain 1 part of copper in 22,000 parts of water. Samples of these were deposited and dried on glass slides. An elongated drop of water, containing about 25 spores per low-power microscopic field, was so placed that one end

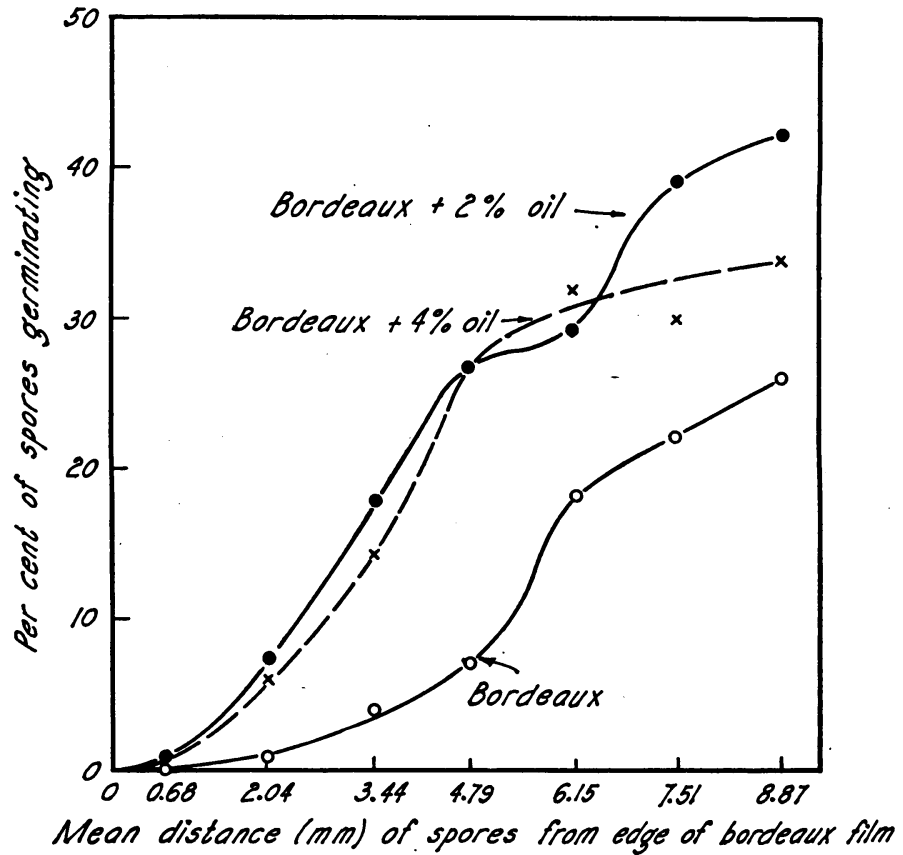

Fig. 2.-Effect of 2 and 4 per cent of oil emulsion on the toxicity of a dried deposit of bordeaux (8-8-100, diluted to contain 1 part of copper in 22,000 parts of water) to spores of Coryneum Beijerinckii lying in water at different distances from the deposit. Bordeaux suppressed germination more than oil-bordeaux at all distances from the deposit.

covered the bordeaux film, the other extending over clean glass for a distance of about 10 millimeters. After 24 hours the percentage of spores germinating was determined in zones located at different distances from the edge of the bordeaux film. According to the data in figure 2, no germination occurred when the spores were located over the film; but as the distance from the film increased, so did germination. The curve for germination over oil-bordeaux films rises more rapidly than that for germination over bordeaux films, indicating, as did the first type of experiment, a somewhat lower toxicity of oil-bordeaux. 
These results represent the level of toxicity of freshly deposited bordeaux films only. That weathering alters the composition of a bordeaux film was recently demonstrated by Wilcoxon and McCallan (11), who found that the first change undergone-the carbonation of the excess lime-was completed within a few hours. The next change, brought about by rain, was the preferential removal of calcium and sulfate. When considerable amounts of these are removed the film becomes proportionately richer in copper. In freshly prepared bordeaux films, copper was only slightly soluble; but as leaching by rain continued, the solubility became greater.

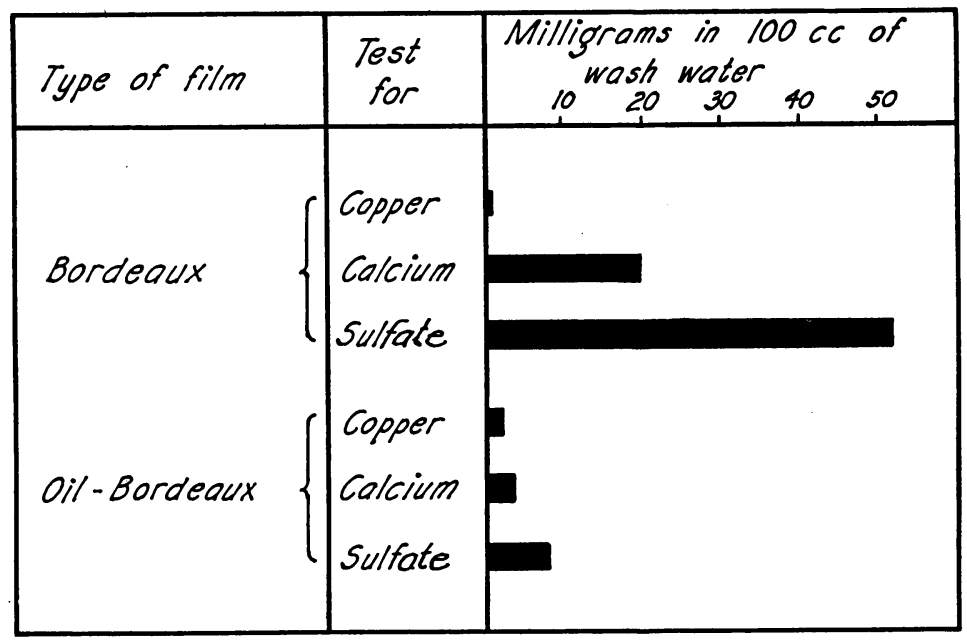

Fig. 3.-Effect of 3 per cent of oil emulsion on the amount of soluble copper, calcium, and sulfate leached from a bordeaux deposit with distilled water applied as "rain." Much of the copper lost from the oil-bordeaux deposit was dissolved in oil that escaped during leaching.

The effect of oil on leaching was therefore studied.Bordeaux (8-8-100) and bordeaux (8-8-100) plus 3 per cent of oil emulsion were deposited in equal quantities in uniform petri dishes. After drying for several days the plates were placed at a slight angle in separate funnels equipped with filter paper. Distilled water was atomized over the surfaces of the films for definite periods. The wash water was collected and analyzed for calcium, sulfates, and copper. In all cases oil retarded the loss of calcium and sulfates, but increased the loss of soluble copper from the films (fig. 3).

A slight turbidity of the wash water from oil-bordeaux film indicated that some material escaping from the film was capable of passing through filter paper. This material proved to be oil, which, upon extraction by 
petroleum ether, proved to contain copper. In fact, most of the copper in the wash water was held by the oil. That the oil dissolves copper from the bordeaux residue was further shown by extracting oil-bordeaux film with anhydrous ether, after thoroughly drying the film in warm air for several hours. Upon evaporating the ether, incinerating the oil, and dissolving the residue in nitric acid, copper was found in considerable amounts.

Though the amounts of copper in wash water from the two types of films was not compared after long-continued leaching, the wash water from oil-bordeaux contained more copper than that from bordeaux at the end of the fourth 1-hour leaching. Obviously, as long as the oil is being removed from the film, the copper dissolved therein will also escape.

Whether or not the oil-dissolved copper renders the wash water more toxic to fungus spores than water from bordeaux films was the next problem. The following are results of a typical experiment: Spores of Sclerotinia fructicola suspended in water which had leached a bordeaux film for 20 minutes and into which had escaped 0.2 p.p.m. of soluble copper, and suspended in water which had leached an oil-bordeaux film for 20 minutes and into which had escaped 0.9 p.p.m. of soluble copper (most of which was dissolved in oil), germinated 73 and 61 per cent respectively, as compared with 89 per cent for the check. Other experiments with spores of Coryneum Beijerinckii indicated still smaller differences between the toxicities of wash waters from the two types of films. Under conditions of these experiments, therefore, the results were such as to suggest that the oil-dissolved copper escaping during leaching imparts little or no additional toxicity to the water. The results of toxicity studies presented in figures 1 and 2 are in accord with this conclusion, inasmuch as unleached oil-bordeaux was slightly less toxic than bordeaux.

\section{DISCUSSION}

The results secured in different phases of this study can now be related to one another. Attention is first directed to the evidence regarding loss of bordeaux from the sprayed surfaces. Wilcoxon and McCallan (11) described the changes that meteorological conditions effect in a bordeaux film after deposition. First, the excess lime is carbonated by action of carbon dioxide; second, rain leaches calcium and sulfates from the film ; and third, the copper increases in solubility and is presumably washed away by rain. Just how rapidly copper is lost from the film by this leaching process is not known. It is probably slower than the loss that results when the bordeaux precipitate, as such, is removed from the surface by the eroding effect of rain. Since the field evidence secured 
in this study does not distinguish between these two types of loss, the role played by oil in preventing loss of both types is not revealed. In laboratory "weathering" tests, however, the oil decreased the loss of calcium and sulfate by leaching. If, as indicated by Wilcoxon and McCallan's studies (11) water-soluble copper increased measurably only after disappearance of considerable calcium and sulfate from the film, then oil probably also delays leaching of copper. As will be remembered, the greater part of soluble copper escaping from oil-bordeaux film during laboratory weathering tests was dissolved in the oil; and the amount lost, in consequence, was limited to the dissolving power of the oil. The oil probably did not increase the amount of water-soluble copper, but very likely decreased it by protecting the bordeaux particles against the leaching effect of rain.

That oil protects the bordeaux from external weathering agencies might also be inferred from the observations that upon drying of an oilbordeaux film the oil droplets broke from their emulsified state and spread over and among the bordeaux particles. The degree to which the bordeaux is oil-coated depends, of course, on the amount of oil used. In this connection one should remember that the tenacity of bordeaux increased with increasing amounts of oil. During the early stages of weathering the increased tenacity may very likely be due to a waterrepellent property conferred upon the bordeaux and the surface by the oil. According to views expressed by Fajan and Martin (3), certain surface-active substances may reduce the tenacity of fungicides because they render the fungicide deposit more wettable by rain. Rendering the fungicide deposit less wettable by the addition of oil may therefore increase tenacity.

As certain emulsifying agents are good wetting agents, their tendency to increase the wetting properties of bordeaux film after it is deposited and dries should not be disregarded. In the present studies the emulsifier present in oil emulsion A was not shown to influence the wettability of the surface by the liquid oil-bordeaux. The reason for this might be that the emulsifier was not an active wetting agent; but a more likely explanation is that the emulsifier was present in the spray in such small amounts. When comparatively large quantities of an organic wetting agent were added to bordeaux, the wetting properties of the spray were increased. Possibly, therefore, an emulsifier that has high wetting powers and is used in amounts greater than in these tests might modify the tendency for oil to increase the tenacity of bordeaux.

Inasmuch as the evidence regarding the effect of oil on both the amount and the distribution of bordeaux deposit is intimately connected with 
the wetting and spreading properties of the liquid, these phenomena must be considered. The wetting property of a liquid is that property which enables the liquid to make stable contact with the surface; the spreading property, on the other hand, determines the extent to which the liquid spreads over the surface by capillary forces $(2,5,9,15)$. The former determines the extent and the persistency of the contact of liquid with surface, and probably depends somewhat on time for maximum expression; the latter determines the final area covered by a given volume of liquid, and definitely depends on time for its maximum expression. In the present studies, the observation that upon hitting the surface the spray droplet did not run off, but occupied a certain area and assumed a definite shape, illustrated the degree to which the liquid wet the surface. If the surface proved nonwettable, the droplets would roll off it without leaving a liquid deposit behind. Drops of bordeaux and oil-bordeaux placed upon a horizontal cellulose nitrate surface did not increase the area they occupied upon hitting the surface-an observation that illustrates the low capillary activity of the sprays on the particular surface. According to these tests, therefore, the addition of oil to bordeaux decreased the wettability of the latter, but did not affect the spreading properties. Hoskins and Ben-Amotz (9) found that an oil-water emulsion containing blood albumin or hemoglobin as the emulsifier would wet beeswax surfaces less easily than the corresponding water solutions of blood albumin or hemoglobin.

When bordeaux and oil-bordeaux were applied to a cellulose nitrate surface, the latter formed drops that occupied less area than those of the former. To cover a given area completely, therefore, a greater number of oil-bordeaux drops must be applied. Assuming that the time in application when the spray began to run from the surface was the point at which the surface was completely covered by liquid, then at this time the film of oil-bordeaux would be thicker than the film of bordeaux, because it requires longer application to produce runoff. Although this view fits the evidence secured, oil-bordeaux may have required a longer period of application than bordeaux to reach the runoff stage because it was more viscous and resisted the tendency of gravity to pull it from the position it occupied upon deposition (15).

From the beginning of application until the runoff stage the amount of deposit increased. When the liquid began to drain from the surface, however, no further increase could occur; on the contrary, the deposit of bordeaux decreased 33 per cent, and that of oil-bordeaux, 27 per cent. It was during the runoff stage that oil was found to improve the distribution of the bordeaux precipitate over the surface. 
Though laboratory tests indicated oil-bordeaux to be slightly less toxic to fungus spores than bordeaux, the field tests revealed no such difference. The former, in fact, gave better control because it remained on twigs over a longer period. Under ordinary conditions, therefore, the slight effect of difference in toxicity is likely to be nullified by the greater effect of increased tenacity.

\section{SUMMARY AND CONCLUSIONS}

For adequate protection of peach trees against the attack of Coryneum Beijerinckii the twigs must be protected by a fungicide throughout the winter. The weather-resisting quality (tenacity) of the fungicide is therefore a determining factor in successful control. The primary purpose of field tests reported herein was to determine how certain added materials, particularly petroleum-oil emulsion, affect the tenacity of bordeaux mixture. The influence of oil on retention, coverage, and toxicity of bordeaux was studied in the laboratory.

When used in sufficient amounts, a dormant-type petroleum oil increased tenacity of bordeaux mixture. Thus 3 or 4 per cent decreased the loss of copper from peach twigs during the winter, but 1 per cent did not. This was true both of a commercial emulsion and of a tank-mix oil emulsion made with blood albumin.

By preventing loss of copper, the oil prolonged the period of protection afforded by a single treatment of bordeaux given in the autumn. In one year when Coryneum Beijerinckii attacked the twigs throughout the winter, the greater protective efficiency of oil-bordeaux was reflected in better control. In another year, however, when attacks of the fungus were confined to early winter, before rains had reduced copper deposits; bordeaux controlled the disease about as effectively as oil-bordeaux.

Neither bentonite ( 0.6 and 2 pounds per 100 gallons of spray) nor cottonseed oil ( 1 per cent) appeared to affect the tenacity of bordeaux or its control of twig infection by Coryneum Beijerinckii.

None of the added materials affected the efficiency with which bordeaux controlled leaf curl (caused by Taphrina deformans). A single autumn application of bordeaux, with or without added materials, effectively reduced the disease.

In certain field tests, oil appeared to affect the amount of bordeaux retained by peach twigs; but the results varied in such a way as to be inconclusive. Laboratory tests, therefore, were performed to determine the retention of bordeaux and oil-bordeaux by vertical surfaces of cellulose nitrate. The sprays were applied with an atomizing apparatus designed to deliver a constant volume of liquid. 
When bordeaux and bordeaux plus 3 per cent of oil emulsion A were applied until the liquid showed signs of running down the surface, the latter deposited an average of 37 per cent more copper than the former. When application was prolonged until approximately $1 \mathrm{cc}$ of liquid had run off the surface (drip stage), the latter deposited 26 per cent more copper than the former. With both types of bordeaux the deposit was greater at the runoff stage than at the drip stage. Between these two stages the copper deposit decreased 33 per cent for bordeaux, 27 per cent for oil-bordeaux.

In trials where the length of time necessary to produce runoff of the liquid from the slide was determined, oil-bordeaux was found to require the longer period.

The effect of petroleum-oil emulsion on coverage, or distribution of bordeaux over the cellulose nitrate surfaces, was observed by examining the area of surface covered by droplets sprayed onto slides. The area covered by a droplet of oil-bordeaux was found to be smaller than that covered by a droplet of bordeaux, as was also the case when equal-sized drops of the two types of bordeaux emitted from a pipette were allowed to fall from a fixed distance onto horizontal cellulose nitrate surfaces. As these drops neither extended nor contracted after deposition and before drying, spreading by capillary forces was apparently not present to any extent. Therefore, upon coming in contact with a surface during application, the area covered by the spray droplets was largely determined by the wetting properties of the liquid. As bordeaux wetted the surface somewhat better than oil-bordeaux, the droplets of this material occupied the larger area; and as fewer were required to cover the surface, less bordeaux than oil-bordeaux was required to produce runoff; hence less was retained by the surface. Difference in viscosity of bordeaux and oil-bordeaux might account, in part, for differences in deposits, although this phase was not studied. Application of the spray at a pressure higher than that employed in these tests might modify this difference in behavior between the two types of bordeaux. Force of impact would force the droplets to spread over areas they do not wet, although they might tend to withdraw from these areas after application ceases.

When application was prolonged until liquid had drained from the surface and the retained precipitate was dried, the oil-bordeaux was found more evenly distributed than the bordeaux. There is, apparently, an explanation: whereas bordeaux drained from the slide in a series of large drops which pursued an uneven course down the slide, leaving heavy and light deposits in their wake, oil-bordeaux drained from the surface as a sheet, leaving a more even deposit. 
Though the laboratory results thus far are not adequate to explain the different problems encountered in the field, they contain suggestions that would account for wide variabilities in deposits between years, or between applications made by different individuals. For example, a tendency to end application at the time the spray begins to drip from the trees might give certain deposits, whereas a tendency to overspray might give different deposits.

The toxicities of the two types of bordeaux to spores of Coryneum Beijerinckii and Sclerotinia fructicola were compared in laboratory studies. Bordeaux mixtures (8-8-100), with and without oil, were of such uniformly high toxicity as to be indistinguishable in this regard. When, however, successive dilutions were made, bordeaux appeared to be somewhat more toxic than oil-bordeaux. Another method of assessing toxicity gave similar results. This method consisted in germinating spores of the two fungi in elongated drops of water, which were placed on slides with one end of the drop resting over a dried film of the fungicide, the other end extending for several millimeters over clean glass. The percentages of spores germinating were determined in zones at different distances from the edge of the fungicide deposit and were greater with oil-bordeaux than with bordeaux.

In artificial weathering tests, 2 per cent or more of oil emulsion was found to reduce the loss of calcium and sulfate from dried bordeaux films. As will be recalled, Wilcoxon and McCallan (11) found that during weathering, a loss of these two constituents was the forerunner of an increase in soluble copper in dried bordeaux films.

In tests of wash water from weathered bordeaux and oil-bordeaux films, more soluble copper was found to escape from the latter than from the former. It was determined, however, that most of the soluble copper was held by oil which escaped during the weathering process. Oil extracted from thoroughly dried oil-bordeaux films by anhydrous ether contained considerable amounts of soluble copper. The presence of this oil-held soluble copper did not appear to increase markedly the toxicity of the wash water to fungus spores. 


\section{LITERATURE CITED}

1. Callan, T., and J. A. R. Henderson.

1929. A new reagent for the colorimetric determination of minute amounts of copper. Analyst 54:650-53.

2. Evans, A. C., and H. Martin.

1935. The incorporation of direct with protective insecticides and fungicides.

I. The laboratory evaluation of water-soluble wetting agents as constituents of combined washes. Jour. Pomol. and Hort. Sci. 13 :261-94.

3. FAJAN, E., and H. MARTIN.

1937. The incorporation of direct with protective insecticides and fungicides. II. The effects of spray supplements on the retention and tenacity of protective deposits. Jour. Pomol. and Hort. Sci. 15:1-24.

4. GreEN, E. L., and M. C. Goldsworthy.

1937. The copper content of residues from sprays containing adjuvants. Phytopathology $27: 957-70$.

5. Hensill, G. S., and W. M. Hoskins.

1935. Factors concerning the deposit of sprays. I. The effect of different concentrations of wetting agents. Jour. Econ. Ent. 28:942-50.

6. HockenYos, G. L., and G. R. IRWIN.

1932. Studies of bordeaux deposition. Phytopathology 22:857-60.

7. Holland, E. B., C. O. Dunbar, and G. M. Gilligan.

1929. Supplements for copper fungicides. Massachusetts Agr. Exp. Sta. Bul. 252:93-112.

8. Horsfall, J. G., J. W. Heuberger, E. G. Sharvelle, and J. M. Hamilton.

1940. A design for laboratory assay of fungicides. Phytopathology 30:545-62.

9. Hoskins, W. M., and Y. BEN-AMOTz.

1938. The deposits of aqueous solutions and of oil sprays. Hilgardia 12(2): 83-111.

10. MagIe, R. O., and J. G. Horsfall.

1936. Relative adherence of cuprous oxide and other copper fungicides. $A b$ stracted in: Phytopathology 26:100-101.

11. Wilcoxon, F., and S. E. A. MCCaLLan.

1938. Weathering of bordeaux mixture. Boyce Thompson Inst. Contrib. 9: 149-59.

12. Wuson, E. E.

1937. The shot-hole disease of stone-fruit trees. California Agr. Exp. Sta. Bul. $608: 1-40$.

13. WILson, E. E.

1939. The effect of petroleum oil emulsion on the fungicidal value of bordeaux mixture. Abstracted in: Phytopathology $29: 828$.

14. Winston, J. R., J. J. Bowman, and W. W. Yothers.

1923. Bordeaux-oil emulsion. U. S. Dept. Agr. Dept. Bul. 1178:1-24.

15. WOODMAN, R. M.

1924. The physics of spray liquid. I. The properties of wetting and spreading. Jour. Pomol. and Hort Sci. 4:38-58.

16. YARWOOD, C. E.

1937. Sulphur and rosin as downy mildew fungicides. Phytopathology 27:931-41. 